\title{
Absence of recovery or dendritic reorganization after neonatal posterior parietal lesions
}

\author{
BRYAN KOLB \\ University of Lethbridge, Lethbridge, Alberta, Canada \\ and \\ JAN CIOE \\ Okanagan University College, Kelowna, British Columbia, Canada
}

\begin{abstract}
Rats with bilateral removal of the posterior parietal cortex on Postnatal Day 5 or 10 were compared behaviorally and neuroanatomically to littermate control rats. The lesioned rats were impaired at the Morris water task but performed the Whishaw reaching task as well as control rats. Although the lesioned rats learned to find the hidden platform nearly as quickly as the control animals, their swim paths were less accurate, reflecting a chronic deficit in orienting the body through space. In contrast to the effects of lesions in the frontal or occipital cortex, posterior parietal lesions were not associated with compensatory dendritic growth in pyramidal cells in adjacent parietal cortex, a result that is consistent with an absence of functional recovery. Also in contrast to the effects of frontal lesions, there was no sex difference in the effects of posterior parietal lesions on either behavior or dendritic arborization in nearby parietal cortex. These results imply that the posterior parietal cortex is fundamentally different in its response to neonatal injury from other neocortical areas such as frontal, motor, or occipital cortex.
\end{abstract}

In a series of studies we have shown that when the cortex of the rat is damaged before 5 days of age, there is a poor behavioral outcome, whereas when it is damaged at about 7-12 days there is substantial recovery of function (e.g., Kolb \& Whishaw, 1989). The behavioral outcomes are correlated with anatomical changes as well: Recovery is associated with increased dendritic arborization and spine density in cortical pyramidal cells, whereas the absence of recovery is associated with decreased dendritic arborization and decreased spine density (see, e.g., Kolb \& Gibb, 1993). The relationship between age, functional recovery, and dendritic changes appears to be true for damage to a broad range of cortical injuries including frontal, motor, and occipital cortex, as well as for hemidecortication (for a review, see Kolb, 1995). One exception to the age and functional recovery relationship appears to be the posterior parietal cortex (PPC), however. Rats with PPC lesions in infancy show surprisingly severe functional deficits relative to adults with similar lesions, regardless of whether their lesions are at 1, 5, 7, or 10 days of age (see, e.g., Kolb, Holmes, \& Whishaw, 1987; Kolb \& Whishaw, 1985). There is a significant temporal gradient, with less severe deficits after lesions at 10 days of age, but even these animals are more impaired than adults with seemingly equivalent lesions. It

This research was supported by a Natural Science and Engineering Council of Canada grant to B.K. and a research grant-in-aid from Okanagan University College to J.C. The authors thank Robbin Gibb and Grazyna for help in the anatomical analysis. Correspondence should be addressed to B. Kolb, Department of Psychology, University of Lethbridge, Lethbridge, AB TIK 3M4, Canada (e-mail: kolb@hg.uleth.ca). was this conundrum that we addressed in the present study.

One explanation for the severity of behavioral effects from infant PPC lesions is that the lesions are not equivalent to those in adulthood. In order to control for this possibility, we altered our lesion parameters in the present study, making much smaller lesions. In addition, since we have found that task difficulty is an important variable in the extent of recovery from early injury, we altered our behavioral procedures in our version of the Morris water task. We have found in unpublished studies that rats with medial frontal lesions at $1-5$ days of age perform dramatically better if the task requires only four trials, rather than eight trials, per day, so in the present study we used the four-trial version.

We also addressed two additional questions. First, we have shown consistently that there are sex-related differences in functional recovery and dendritic change after medial frontal lesions in infancy (e.g., Kolb \& Stewart, 1995). We found no such sex differences after occipital lesions (Kolb, Ladowsky, Gibb, \& Gorny, 1995), so we were interested in determining whether sex played a role in recovery from PPC lesions. Second, since we have consistently found a relationship between functional recovery and dendritic changes, we wondered whether the failure to find good functional recovery after neonatal PPC lesions reflected an absence of dendritic growth. Certainly, if dendritic growth were found after the early PPC lesions, the validity of our dendrite-function correlation would be questionable!

Rats were given PPC lesions on Postnatal Day 5 or 10. They were tested on two behavioral measures, the Mor- 
ris water task and the Whishaw reaching task, in adulthood. We chose to use these behavioral measures for two reasons. First, adult rats with PPC lesions are impaired at the Morris task. Second, rats with adult PPC lesions are not impaired at the reaching task. Since we had previously found that neonatal PPC lesions were associated with novel deficits on cognitive tasks on which rats with adult PPC lesions were not impaired (Kolb \& Whishaw, 1985), we wondered whether they would also show deficits on a motor task. At the conclusion of behavioral testing, the animals' brains were prepared with a modification of the Golgi-Cox method (Gibb \& Kolb, 1998), and the cortical pyramidal cells in Zilles's (1985) area Par 1 were analyzed for dendritic arborization and spine density. We have found these cells to be especially labile after injury from medial frontal, motor, or occipital lesions at Day 10, so it seemed reasonable to examine these cells.

\section{METHOD}

\section{Subjects}

We used 29 rats, derived from the Charles River Long-Evans strains, which were divided into three groups: (1) control ( $4 \mathrm{M}, 4 \mathrm{~F})$, (2) PPC lesions performed at Postnatal Day 5 (P5; $6 \mathrm{M}, 3 \mathrm{~F}$ ), and (3) lesions performed at Postnatal Day 10 (P10;6 M, 6 F). The animals came from two litters ( $n \mathrm{~s}=13$ and 16$) ; 4$ controls $(2 \mathrm{M}, 2 \mathrm{~F}$ ) came from each litter. One male control animal died from unknown causes prior to weaning. The surviving animals were same-sex housed in groups of 3 in stainless steel hanging cages and maintained on ad-lib water and a 12:12-h light:dark schedule throughout the experiments. The animals were on ad-lib food throughout except during the Whishaw reaching task. The control and lesioned rats were tested in the behavioral tasks in the order described below. Testing began when the animals were about 90 days old. The brains were prepared for histological analysis when the rats were about 200 days old.

\section{Surgical and Anatomical Procedures}

The animals were anesthetized by cooling them in a Thermatron cooling chamber until their rectal body temperatures were in the range of $18^{\circ}-20^{\circ} \mathrm{C}$. In preliminary studies, we had identified the location of presumptive posterior parietal cortex, which is roughly Krieg's area 7 (see Kolb \& Walkey, 1987), of the young brain to lie somewhat more anterior at 5-10 days than it does in adulthood. (This presumably reflects the anterior-posterior maturation gradient of the cortex; Bayer \& Altman, 1987). The location of the presumptive posterior parietal area was determined by dividing the distance between the bregma and lambda into thirds. The skull over the middle third was removed from 1-mm lateral to the sagittal fissure to a point equal to anterior-posterior length. Accordingly, a hole was cut in the skull from which the exposed posterior parietal cortex was extracted by gentle suction, taking care not to open the ventricle. The animals were sutured with silk thread as soon as the operation was complete. The normal control animals were anesthetized, the skin incised, and then closed with silk suture. The control animals were littermates of the experimental animals. Following suturing, the animals were warmed and once all the animals had returned to about $37^{\circ} \mathrm{C}$, they were returned to their mother. The animals were checked daily to ensure that their wounds were healing quickly and that they were all nursing. (In young rats the milk can be seen in the stomach until the fur grows over at about 2 weeks.)

Following the conclusion of behavioral testing ( 200 days of age), the animals were given an overdose of sodium pentobarbital and intracardially perfused with $0.9 \%$ saline. The brains were removed and weighed before being immersed whole in $20 \mathrm{ml}$ of Golgi-Cox solution. The brains were left in the solution for 12 days before being placed in a $30 \%$ sucrose solution for 2 days, cut on a Vibratome at $200 \mu \mathrm{m}$, and developed using a procedure described by Gibb and Kolb (1998). Layer II/III pyramidal cells in Zilles's area Par 1 were traced using a camera lucida at $250 \times$. In order to be included in the data analysis, the dendritic trees of pyramidal cells had to fulfill the following criteria: (1) The cell had to be well impregnated and not obscured with blood vessels, astrocytes, or heavy clusters of dendrites from other cells; and (2) the apical and basilar arborizations had to appear to be largely intact and visible in the plane of section. The cells were drawn and analyzed using two different procedures. In the first, each branch segment was counted and summarized by branch order using the procedure of Coleman and Riesen (1968). Branch order was determined for the basilar dendrites in such a way that branches originating at the cell body were first order; after one bifurcation, second order; and so on. Branch order was determined for the apical dendrites in such a way that branches originating from the primary apical dendrite were first order, and so on. A Sholl (1956) analysis of ring intersections was used to estimate dendritic length. The number of intersections of dendrites with a series of concentric spheres at $20-\mu \mathrm{m}$ intervals from the center of the cell body was counted for each cell. Total dendritic length (in micrometers) can be estimated by multiplying the number of intersections by 20 .

Cells were chosen by locating the parietal cortex (Zilles's Par 1) at the level of the anterior commissure and then by drawing each cell in the section that met the criteria listed above. This region is distinct in Golgi-stained sections, and normally it is possible to obtain sufficient cells from two adjacent sections. Ten cells were drawn in each hemisphere of each rat. The statistical analyses were done by taking the mean of the measurements on the 10 cells for each subject.

Spine density was measured from one apical dendritic branch in the terminal tuft, one secondary apical branch beginning about $50 \%$ of the distance between the cell body and terminal tuft, one basilar terminal branch (which was always a fourth-order terminal branch), and one secondary basilar branch. Spine density measures were made from a segment greater than $10 \mu \mathrm{m}$ in length, and usually about $50 \mu \mathrm{m}$. The dendrite was traced $(1,000 \times)$ using a camera lucida drawing tube and the exact length of the dendritic segment calculated by placing a thread along the drawing and then measuring the thread length. Spine density was expressed as the number of spines per $10 \mu \mathrm{m}$. No attempt was made to correct for spines hidden beneath or above the dendritic segment, so the spine density values are likely to underestimate the actual density of the dendritic spines.

\section{Morris Water Task}

The method followed in the Morris water task (Morris, 1981) is virtually identical to that used by Sutherland, Whishaw, and Kolb (1983). The maze consisted of a circular pool (diameter, $1.5 \mathrm{~m}$; height, $45 \mathrm{~cm}$ ), the inside of which was painted white and filled to a height of $25 \mathrm{~cm}$ with approximately $18^{\circ} \mathrm{C}$ water in which $1 \mathrm{~L}$ of instant powdered milk was dissolved. A clear Plexiglas platform $(11 \times 12 \mathrm{~cm})$ was present inside the pool; its top surface was $1 \mathrm{~cm}$ below the surface of the water, and thus the platform was invisible to a viewer inside the pool.

A trial consisted of placing a rat by hand into the water, facing the wall of the pool, at one of four starting locations (north, south, east, or west) around the pool's perimeter. Within each daily block of four trials, each rat started at the four starting locations, but the sequence of locations was randomly selected.

The behavioral testing was conducted on 10 consecutive days, with each rat receiving four trials per day. If on a particular trial a rat found the platform, it was permitted to remain on the platform for $10 \mathrm{sec}$. A trial was terminated if a rat failed to find the platform after $90 \mathrm{sec}$. At the end of a trial, the rat was returned to a holding cage, and approximately 5 min elapsed before beginning the next 
trial. The latency to find the platform was timed by an experimenter standing by the pool's edge. The route traversed by each rat was traced by a second observer using pencil and paper. An error was scored if the rat's path deviated from a $20-\mathrm{cm}$-wide channel going directly from the start point to the platform. The hidden platform was kept in a constant location for all 10 trial blocks.

\section{Whishaw Reaching Task}

The Whishaw reaching task was adapted from the method developed by Whishaw and colleagues (Whishaw, O'Connor, \& Dunnett, 1986). Each animal was food deprived to $85 \%$ body weight for the training and testing. The animals were placed in the test cages (10 $\times 18 \times 10 \mathrm{~cm}$ high) with floors and fronts constructed of $2-\mathrm{mm}$ bars, $9 \mathrm{~mm}$ apart from edge to edge. A 4-cm-wide $\times 5$-cm-deep tray containing $45-\mathrm{mg}$ food pellets (chicken feed) was mounted in the front of each box. The rats were required to extend a forelimb through the gap in the bars and then grasp and retract the food. The tray was mounted on runners and was retracted $0.5 \mathrm{~cm}$ from the cage so that the rats could not scrape the food into the cage. If the animal attempted to rake the pellet out of the tray, the pellet would fall irretrievably through the gap. An attempt was scored only when the rat reached into the tray and touched the food pellet. If it reached into the tray without touching a pellet, no attempt was scored. Animals were trained for a minimum of 10 days, by which time their performance had asymptoted.

\section{RESULTS}

\section{Anatomical Findings}

Brain and body weights. Overall, the brain weights of the prenatally lesioned animals were less than control weights (Table 1); these reductions, however, were smaller than those found in Kolb et al. (1987), presumably reflecting the smaller lesions in the present study. Since brain weight is sexually dimorphic, a two-way analysis of variance (ANOVA) for sex and lesion was conducted; this showed significant main effects of lesion $[F(2,22)=$ $6.047, p=.008]$ and of $\operatorname{sex}[F(1,22)=17.6, p=.0004]$, but not an interaction $[F(2,22)=0.42, p=.66]$.

A comparison of body weights was also done on these animals (the body weight was unavailable for 1 of the female $P 5$ animals because its weight had been incorrectly recorded). A two-way ANOVA demonstrated a significant difference between male $(M=448 \pm 11.8 \mathrm{~g})$ and female $(M=314 \pm 3.9 \mathrm{~g})$ body weights, as was expected $[F(1,21)=32.705, p=.0001]$. However, there was no main effect of lesion $[F(2,21)=1.271, p=.3013]$, nor was there an interaction $[F(2,21)=0.957, p=.4002]$. Hence, although the lesions affected the brain weights, they did not produce systematic differences in body weights (Table 1).

Gross inspection of the brains. Visual inspection of the lesions revealed that, with the exception of 1 P 10 parietal rat, there was a clearly visible lesion cavity in the PPC (for photographs of similar brains, see Kolb et al., 1987). The lesion cavity differed considerably from those seen in the brains of rats receiving parietal lesions in adulthood. Adult lesions leave a clear cavity that does not change much in size from the day of surgery until death. In contrast, brains with infant lesions have more dimple shaped cavities that appear to have collapsed in, leaving a smaller hole (Figure 1). (For a more detailed discussion of this topic and illustrative photographs taken immediately after surgery and in adulthood, see Kolb et al., 1987.) It is clear from Figure 1, however, that the lesions were bigger than the cavity size. The crosssectional area of the cortex was clearly reduced, a phenomenon we have quantified elsewhere (e.g., Kolb et al., 1987).

We note that the lesions formed cavities even though, at the time of surgery, the white matter was not invaded. It appears that the external capsule is simply too fragile at this age to survive the removal of the overlying gray matter.

Examination of the Golgi-stained sections revealed that the lesions spared the cingulate cortex, but for most rats there was some incursion into the somatosensory cortex (Figure 1). In many animals the hippocampus was shifted posteriorly and often pushed up into the lesion cavity, especially in the anterior sections. The hippocampi themselves were not damaged, however, and the cell lamination appeared essentially normal, although somewhat distorted in appearance. Since the sections were stained for Golgi processing, it was not possible to examine the thalamus in much detail.

In order to compare the lesion extent in the P5 and P10 animals, we counted the total number of brain sections for each brain. (We kept and mounted every section.) We then identified the first section with the anterior commissure crossing the midline in each brain. This provided a marker for the anterior-posterior location of the lesion. Finally, we identified the first and last sections with lesions. This allowed us to demarcate the lesion boundaries.

The results showed that the fixed brains were approximately equivalent in length $(12 \mathrm{~mm})$, and the anterior commissure was in virtually the same section in every brain (Table 2). Measurements of the beginning and ending of the lesions did show a small difference at the two lesion ages, however. The P10 lesion began $0.9 \mathrm{~mm}$ further anterior and the P5 lesion ended $0.4 \mathrm{~mm}$ further posterior. Student $t$ tests showed that the anterior difference was significant $[t(19)=8.5, p<.01]$ but that the posterior difference was not significant $[t(19)=1.85, p=.19]$.

Table 1

Summary of Brain and Body Weights

\begin{tabular}{|c|c|c|c|c|c|c|c|c|c|c|c|c|}
\hline \multirow[b]{3}{*}{ Group } & \multicolumn{6}{|c|}{ Brain Weight } & \multicolumn{6}{|c|}{ Body Weight } \\
\hline & \multicolumn{3}{|c|}{ Male } & \multicolumn{3}{|c|}{ Female } & \multicolumn{3}{|c|}{ Male } & \multicolumn{3}{|c|}{ Female } \\
\hline & $M$ & $S E M$ & $n$ & $M$ & SEM & $n$ & $M$ & $S E M$ & $n$ & $M$ & $S E M$ & $n$ \\
\hline Control & 2.13 & .04 & 3 & 2.00 & .03 & 4 & 450 & 18 & 3 & 325 & 10 & 4 \\
\hline P10 parietal & 2.02 & .05 & 6 & 1.92 & .02 & 6 & 450 & 18 & 6 & 309 & 7 & 6 \\
\hline P5 parietal & 2.01 & .03 & 6 & 1.85 & .01 & 3 & 394 & 36 & 6 & 299 & 6 & 2 \\
\hline
\end{tabular}



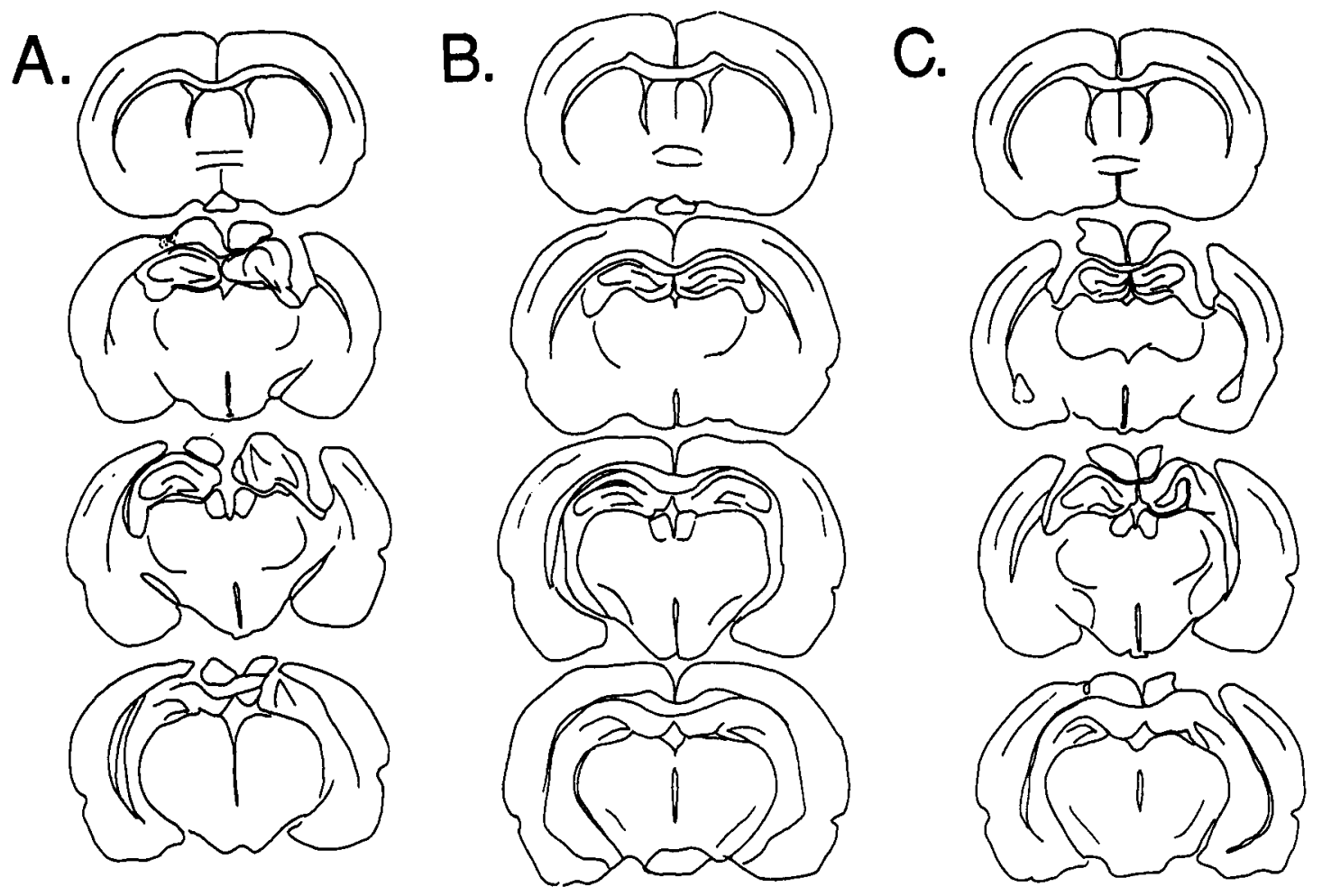

Figure 1. Illustrations showing coronal sections through the brains of representative animals in the P5 (A), control (B), and P10 (C) groups. It is evident that although the lesion cavities were small, the cross-sectional area of the remaining cortex was significantly more reduced than would have been predicted from the cavity size.

In sum, the lesions were largely confined to the region of the PPC, although there was a slight incursion into the posterior part of the somatosensory cortex and into the anterior part of the occipital cortex. The lesions were roughly equivalent at the two ages.

Dendritic arborization: Branch analysis. Parietal lesions at either P5 or P10 significantly reduced not only the total number of apical branches but also the number of branches at each branch order in adulthood. There was no lesion effect for the basilar dendritic branching, however (Figures 2 and 3; Tables 3 and 4).

A two-way ANOVA revealed a significant main effect of lesion $[F(2,50)=6.0, p=.005]$ but no effect for sex $[F(1,50)=0.661, p=.42]$ or interaction $[F(2,50)=$ $0.696, p=.50]$ on the mean total number of apical

Table 2

Summary of Mean Brain Measures (in Millimeters)

\begin{tabular}{lcccc}
\hline \multicolumn{1}{c}{ Group } & Length & $\begin{array}{c}\text { Anterior } \\
\text { Commissure }\end{array}$ & First Lesion & Last Lesion \\
\hline Control & 12.1 & 5.7 & & \\
P10 parietal & 11.9 & 5.7 & $5.4^{*}$ & 9.4 \\
P5 parietal & 11.9 & 5.8 & 6.3 & 9.8 \\
\hline
\end{tabular}

Note-Numbers refer to mean length of the brain (length), distance from the frontal pole to the anterior commissure, distance from the frontal pole to the first section with a lesion (First Lesion), and distance from the frontal pole to the last section with a lesion (Last Lesion). *The first section with a lesion was significantly $(p<.05)$ more anterior in the brains with parietal lesions on Postnatal Day 10. branches. A post hoc analysis (Fisher LSD, $p<.05$ ) demonstrated a significant reduction in the mean total number of apical branches for both P5 and P10 animals compared with controls; the P10 lesion also significantly reduced the number of apical branches compared with the P5 lesion (Figure 2). Similar analyses showed significant differences between the control and P5 animals for Branch Orders 1, 4, 5, and 6+ (Figure 2). There were also significant differences between the controls and P10 animals at Orders $2,3,4,5$, and 6+. The P5 and P10 animals differed at Orders 1 and 3 .

A two-way ANOVA on the mean total number of basilar branches showed no effect of lesion $[F(2,50)=2.57$, $p=.08]$, no effect of $\operatorname{sex}[F(1,50)=1.98, p=.17]$, and no interaction $[F(2,60)=0.688, p=.51]$.

Sholl analysis of dendritic length. Parietal lesions at $P 5$ reduced the total length of both the apical and the basilar dendrites; parietal lesions at $P 10$ reduced only the apical length (Table 5). A two-way ANOVA on apical Sholl intersections revealed a significant main effect of lesion $[F(2,50)=5.74, p=.006]$ but no effect of sex $[F(1,50)=0.307, p=.58]$ and no interaction $[F(2,50)=$ $0.802, p=.45]$. A post hoc analysis (Fisher LSD, $p<$ .05 ) demonstrated a significant reduction in the mean total length for only the $\mathrm{P} 10$ animals relative to controls.

A two-way ANOVA on basilar Sholl crossings revealed a significant main effect of lesion $[F(2,50)=4.2, p=.02]$ but no effect of $\operatorname{sex}[F(1,50)=2.47, p=.12]$ and no 

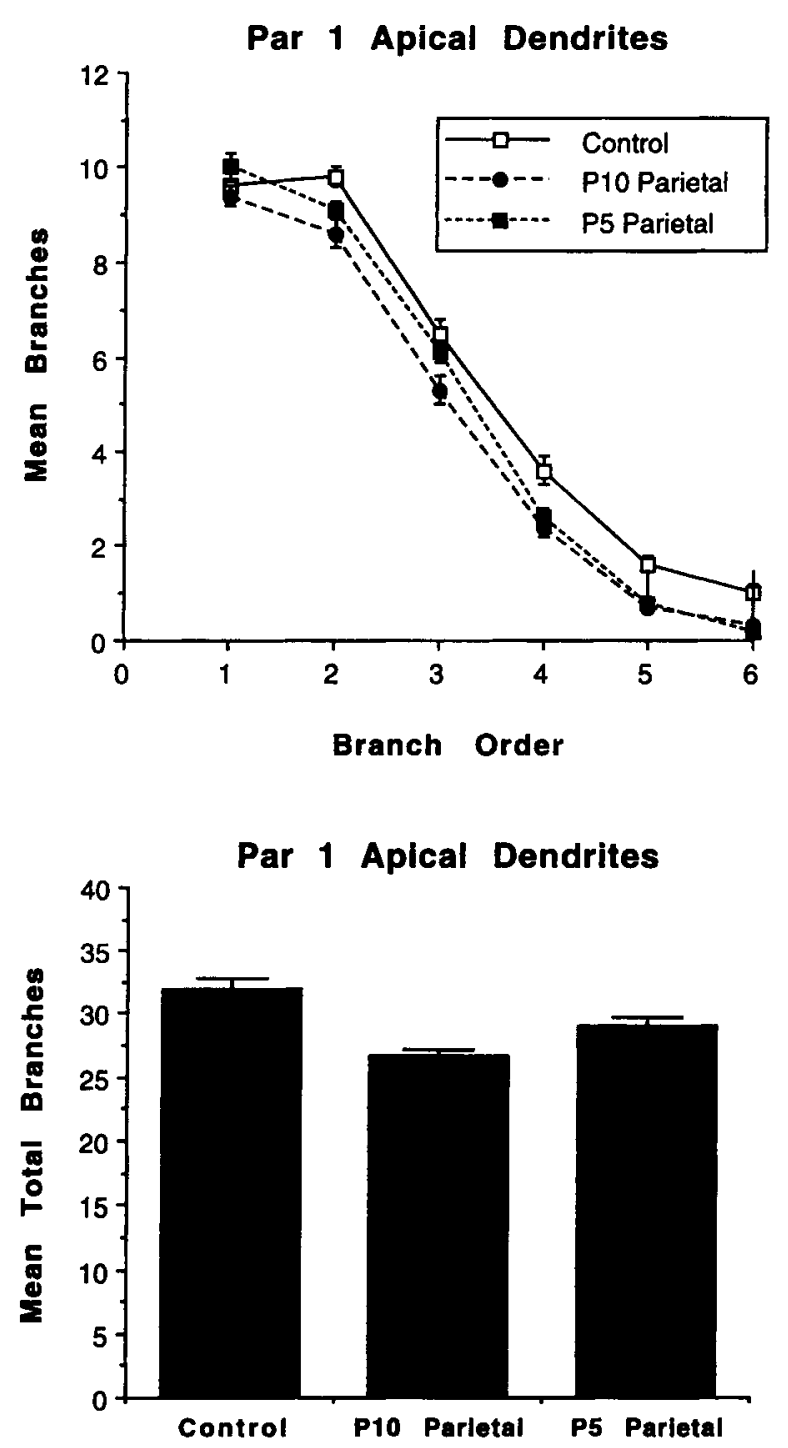

Figure 2. Summary of the dendritic arborization in the apical fields of the Par 1 Layer III pyramidal neurons. There was a significant reduction in total branching in both lesion groups relative to controls. This can be seen at Branch Orders 2-6+. Numbers reflect means and standard errors.

interaction $[F(2,50)=0.068, p=.93]$. A post hoc analysis (Fisher LSD, $p<.05$ ) demonstrated a significant reduction in the P5 group relative to both other groups, which did not differ from each other.

Spine density. Both the P5 and the P10 lesions dramatically reduced spine density on the terminal tips of the apical fibers, on the oblique measure of the apical fibers, and on the terminal tips of the basilar fibers relative to control values. There were no significant differences, however, for the oblique measure of the basilar fibers (Tables 3 and 4).

Two-way ANOVAs for each of these measures revealed significant main effects for the lesions on all but the secondary branch measure of the basilar fibers. There were no significant main effects of sex, and no interaction. Accordingly, one-way ANOVAs were conducted on each measure. Post hoc analysis (Fisher LSDs, $p<.05$ ) revealed significantly lower spine densities for both P5 and P10 animals relative to the controls on the apical and basilar terminal tips as well on the oblique apical fibers. Moreover, the P10 animals had fewer spines on the apical terminal tips relative to the P5 animals.

\section{Behavioral Findings}

Morris water task. The control rats in this study performed much like those described in detail elsewhere (Sutherland et al., 1983). When initially placed in the milk tank, the normal control rats swam over a wide area until they accidentally bumped into the hidden platform. Performance improved rapidly on successive trials until it
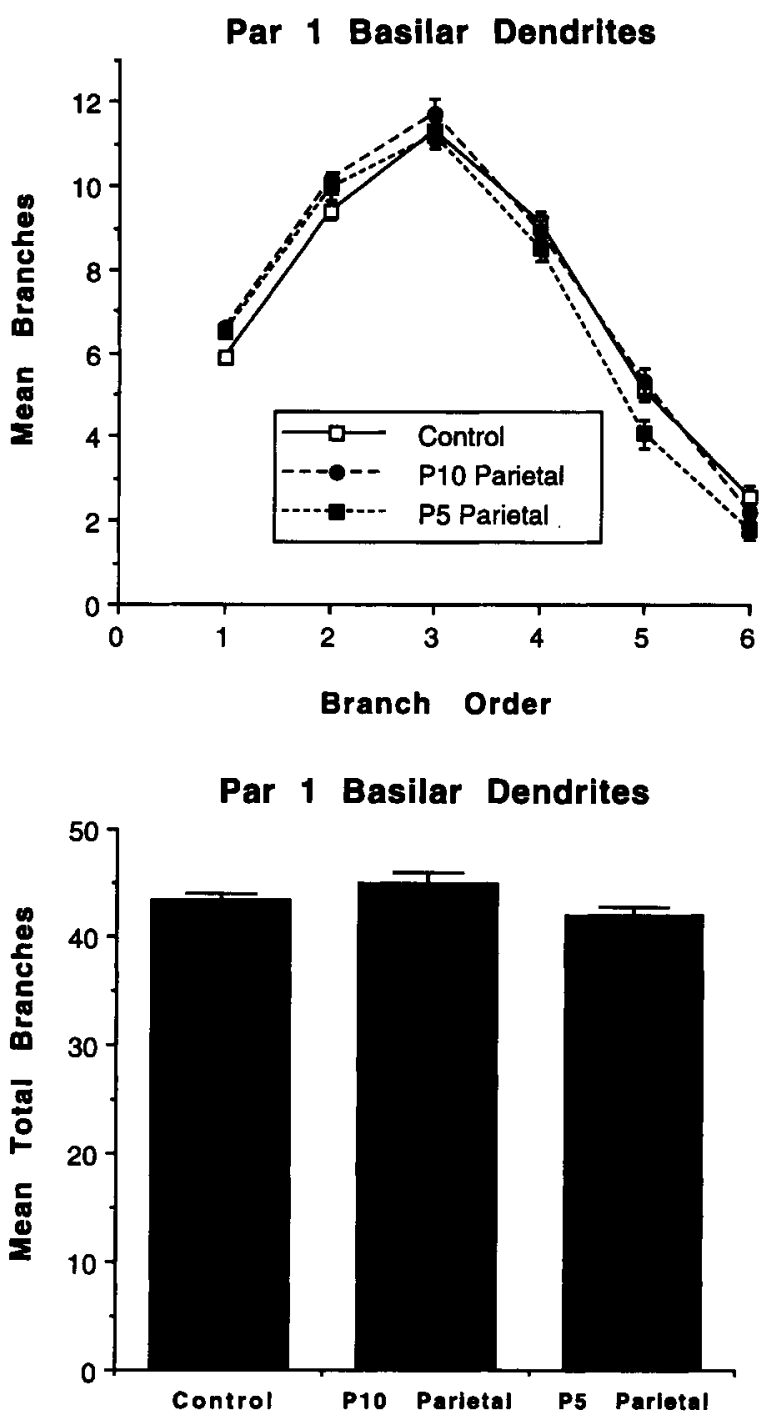

Figure 3. Summary of the dendritic arborization in the basilar fields of the Par 1 Layer III pyramidal neurons. There was no lesion effect. Numbers reflect means and standard errors. 
Table 3

Summary of Apical Branches and Dendritic Spine Density

\begin{tabular}{|c|c|c|c|c|c|c|}
\hline \multirow[b]{2}{*}{ Group } & \multicolumn{2}{|c|}{ Branches } & \multicolumn{2}{|c|}{ Terminal Tip } & \multicolumn{2}{|c|}{ Secondary } \\
\hline & $M$ & $S E M$ & $M$ & $S E M$ & $M$ & $S E M$ \\
\hline ontrol & 32.0 & 0. & 6.2 & .05 & 10.5 & .26 \\
\hline P10 parietal & 26.6 & $0.6^{*}$ & 5.0 & $.10^{*}$ & 9.5 & $.10^{*}$ \\
\hline P5 parietal & 29.1 & $0.7^{*}$ & 5.4 & $.06^{*} \dagger$ & 9.3 & $.09 *$ \\
\hline
\end{tabular}

*Differed significantly from the control group, $p<.05$. † Differed significantly from the $\mathrm{P} 10$ group, $p<.05$.

Table 4

Summary of Basilar Branches and Dendritic Spine Density

\begin{tabular}{|c|c|c|c|c|c|c|}
\hline \multirow[b]{2}{*}{ Group } & \multicolumn{2}{|c|}{ Branches } & \multicolumn{2}{|c|}{ Terminal Tip } & \multicolumn{2}{|c|}{ Secondary } \\
\hline & $M$ & $S E M$ & $M$ & SEM & $M$ & $S E M$ \\
\hline ontrol & 43.4 & 0.6 & 6.6 & .05 & 8.4 & .14 \\
\hline Pl0 parietal & 45.0 & 1.0 & 6.2 & $.11^{*}$ & 8.4 & .12 \\
\hline P5 parietal & 42.0 & 0.9 & 6.3 & $.09^{*}$ & 8.6 & .08 \\
\hline
\end{tabular}

*Differed significantly from the control group, $p<.05$.

Table 5

Summary of Sholl Intersections

\begin{tabular}{lccccl}
\hline & \multicolumn{2}{c}{ Apical } & & \multicolumn{2}{c}{ Basilar } \\
\cline { 2 - 3 } \cline { 5 - 6 } Group & $M$ & $S E M$ & & $M$ & $S E M$ \\
\hline Control & 76.1 & 1.8 & & 97.4 & 1.8 \\
P10 parietal & 67.2 & $1.6^{*}$ & & 96.3 & 2.5 \\
P5 parietal & 71.8 & 2.1 & & 88.5 & $2.2^{*} \dagger$ \\
\hline
\end{tabular}

Note- $M \mathrm{~s}$ and $S E M \mathrm{~s}$ are for the total number of intersections of dendritic segments across the concentric rings, each of which was separated by $20 \mu \mathrm{m}$. *Differed significantly from the control group ( $p<$ $.05)$. Differed significantly from the P10 group $(p<.05)$.

reached asymptote around $5 \mathrm{sec}$ by Trial Block 5 (20 trials). Rats with Day 5 and Day 10 prenatal parietal lesions showed an equivalent impairment at the task (Figure 4).

Because previous work has shown a sex difference as a result of frontal cortical lesions (Kolb \& Stewart, 1995), a two-way ANOVA with lesion and sex was performed using the total escape latency measure. This analysis showed a significant effect of lesion $[F(2,27)=$ $6.47, p=.0051]$ with a trend toward a sex difference $[F(1,27)=3.673, p=.0659]$ but no interaction $[F(2,27)=$ $0.837, p=.44]$. A closer examination of the data over trial blocks suggested that the sex difference became apparent only after several trial blocks. Accordingly, the total escape latency scores over Trial Blocks 4-10 were examined using a two-factor ANOVA (Figure 5). This analysis revealed a highly significant main effect for both lesion $[F(2,27)=10.364, p=.0005]$ and sex $[F(1,27)=$ $13.551, p=.001]$, but no interaction $[F(2,27)=2.383$, $p=.11]$. A one-way ANOVA for females showed only that the total latency for Trial Blocks 4-10 differed significantly by lesion condition, and Fisher LSDs $(p<.05)$ showed longer escape latencies for both the P10 and P5 animals relative to controls; the lesioned animals did not differ significantly across conditions. A similar analysis with the males showed a longer latency only for the P10 animals relative to the controls.
The error measure is a more sensitive index of the actual behavior of the animals in the Morris water task and showed more errors for both lesioned groups compared with the control animals, as well as a reliable sex difference (Figure 6).

In order to deal with the variability of the error measure scores, the data were analyzed over five trial blocks (each block included eight trials). A two-way ANOVA on the mean total error measure revealed both a significant main effect for lesion $[F(2,27)=21.884, p=.0001]$ and sex $[F(1,27)=5.427, p=.03]$, but no interaction $[F(2,27)=$ $3.091, p=.0618]$. Compared with the control animals, the P10 animals showed significantly more errors (Fisher LSDs, $p<.05$ ) on all but the first two blocks, whereas the P5 animals showed more errors on all but the first block

\section{Latency by Trial Block}
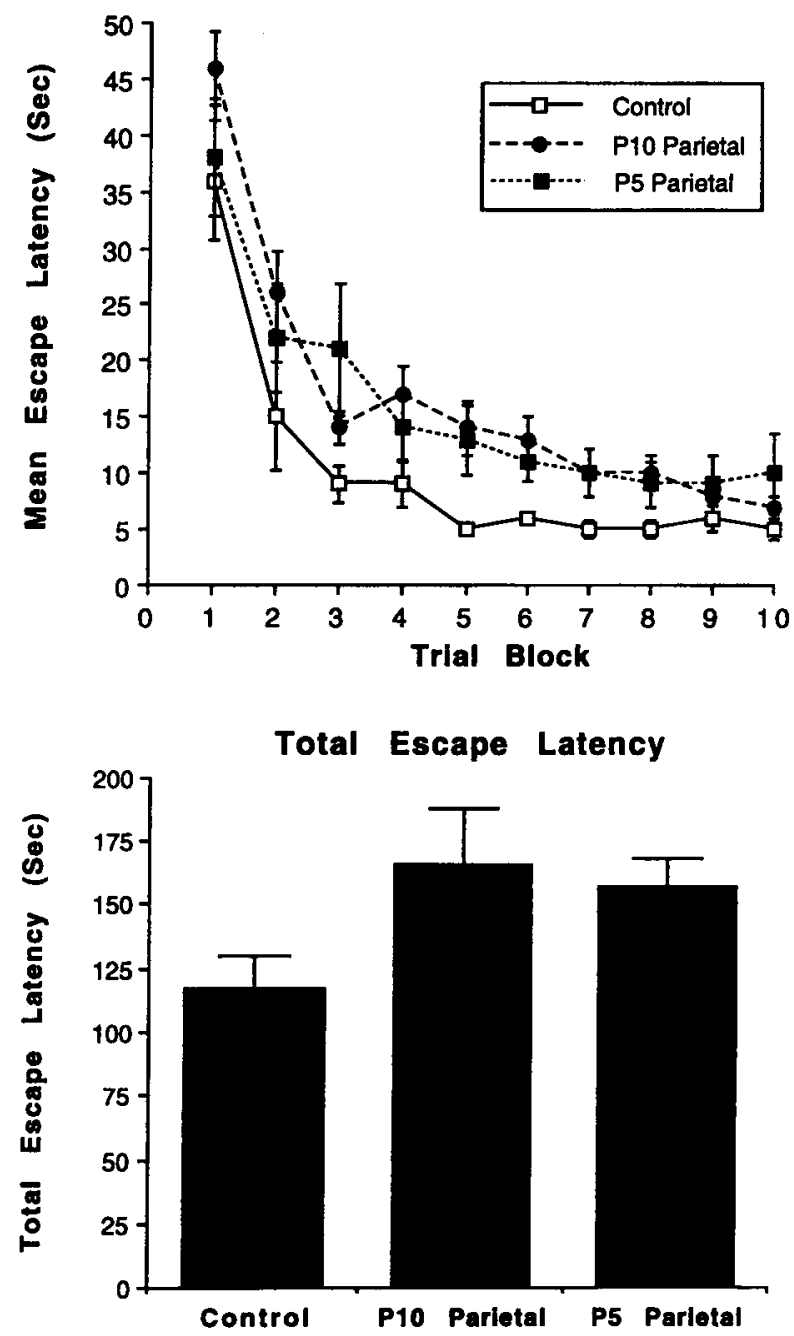

Figure 4. Summary of escape latencies in the Morris water task. Both lesion groups had longer latencies than controls. The top graph shows the performance over trial blocks; the bottom graph shows the total escape latency summed across all trial blocks. Numbers reflect means and standard errors. 


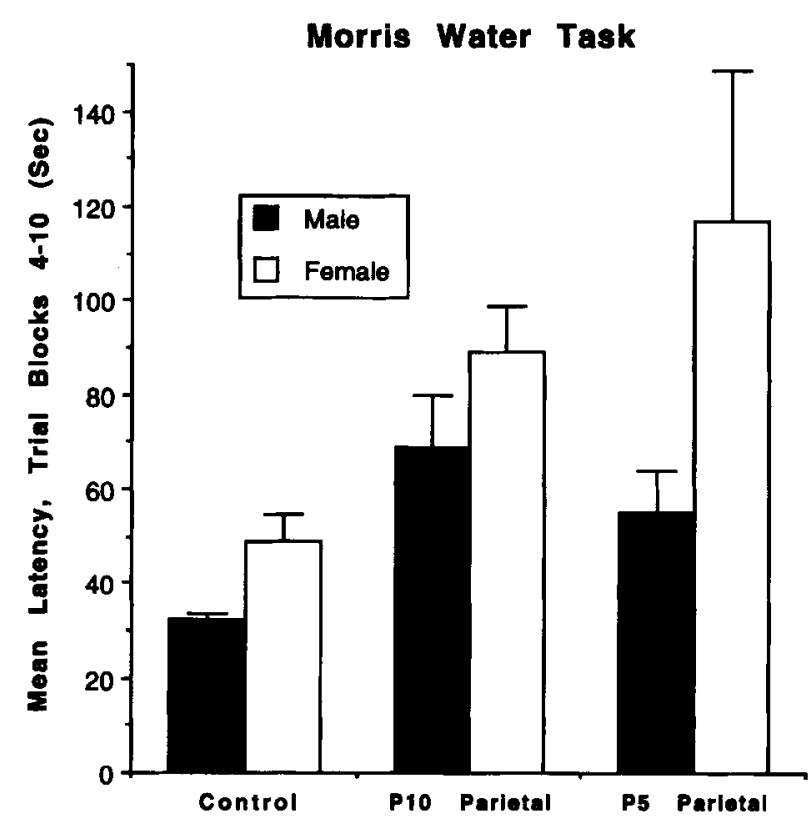

Figure 5. Summary of escape latencies in the Morris water task on Trial Blocks 4-10, with sex as a factor. There was a significant sex effect in that males were superior to females.

(Figure 6). Post hoc analysis of both males and females revealed that the P10 and P5 animals had significantly more total errors than the control animals (Figure 6). The female controls had significantly more total errors than the male controls $[F(1,11)=6.4918, p=.0271]$; there were no sex differences for the $\mathrm{P} 10[F(1,9)=0.6818, p=$ $.4303]$ or $\mathrm{P} 5$ animals $[F(1,8)=0.1287, p=.1287]$.

Whishaw reaching task. Control rats learned to reach quickly and asymptoted at about $40 \%$ accuracy. Both lesion groups learned the task as quickly as controls and were indistinguishable from control animals. An ANOVA indicated no significant differences among the mean scores for the three groups $[F(2,31)=0.06, p=.94]$.

\section{DISCUSSION}

Rats with PPC lesions in infancy have chronic deficits in spatial navigation and do not show any compensatory changes in dendritic arborization. Several issues emerge from these findings, and we consider each separately.

\section{Functions of the PPC}

The PPC of primates is principally composed of regions forming the dorsal visual stream. The function of this stream is controversial (Milner \& Goodale, 1995; Ungerleider \& Mishkin, 1982), but there is little doubt that it is involved in the guidance of movements in space. In the highly visual primate, this is especially true for the control of limb, hand, and eye movements. Limb movements are not under visual control in the rat (Whishaw \& Tomie, 1989), and damage to the PPC does not affect skilled reaching behavior (e.g., the present study). It seems likely, however, that body orientation is visually controlled, especially with respect to orienting the nose and vibrissae relative to objects as well as to directing navigation of the body to objects in space. Rats with PPC lesions in adulthood are impaired at a variety of visuospatial tasks (see, e.g., DiMattia \& Kesner, 1988a, 1988b; Kesner, Farnsworth, \& DiMattia, 1989; Kolb, Buhrmann, McDonald, \& Sutherland, 1994; Kolb, Sutherland, \& Whishaw, 1983; Kolb \& Walkey, 1987; see also other papers in this issue). The persistent deficit in the Morris water task is instructive since we have shown consistently that rats with PPC lesions can learn the Morris task

\section{Errors by Trlal Block}
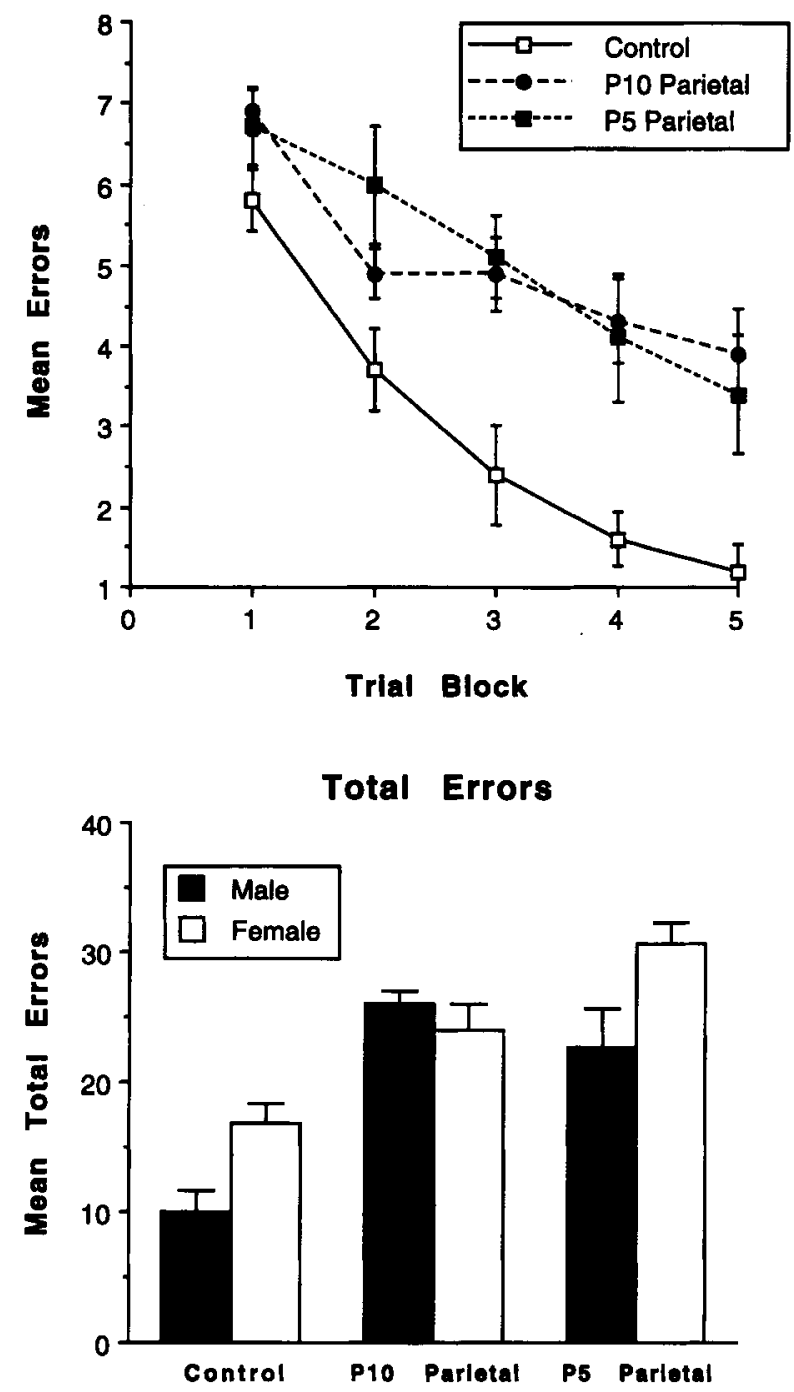

Figure 6. Summary of errors in the Morris water task. The rats with lesions were significantly worse in their swim trajectories and rarely swam directly to the platform. The top graph illustrates performance across trial blocks. The bottom graph illustrates the total error score, with sex as a factor. Female control and $P 5$ lesion animals performed significantly worse than males in the same treatment groups. 
or variants of it, but they are never able to direct their locomotion accurately to a point in space. This deficit is unaffected by extensive practice or preoperative training. It appears that the animals simply cannot direct their locomotion accurately in space without the PPC.

\section{Recovery From PPC Lesions}

Rats with lesions of the motor or medial frontal cortex show impressive functional recovery after lesions at 7-10 days of age and a complete absence of recovery after similar lesions at $1-5$ days of age (for a review, see Kolb, 1995). In contrast, rats with PPC lesions at any age show deficits and rats with lesions at 1-5 days are markedly worse on parietal-sensitive tasks than adults with similar lesions (see, e.g., Kolb et al., 1987; Kolb \& Whishaw, 1985). In the present study, we tried to make the behavioral task easier, and we made smaller lesions than in our earlier studies; there was still little functional recovery. The absence of recovery after neonatal PPC but not neonatal frontal lesions is puzzling. It appears that some aspect of PPC structure and/or connectivity is not replaceable, even under what normally would be optimal conditions. Although highly speculative, we must also note that people with developmental abnormalities in the PPC show persistent dyslexia, and it is tempting to draw a parallel with the poor recovery in dyslexic children and our infant rats. The fact that children with massive lesions to Broca's or Wernicke's area show functional recovery, yet those with ectopias in the PPC do not, is puzzling (for a review, see Galaburda, 1993).

One of the questions that we asked in the present study was whether the devastating morphological consequences of neonatal PPC lesions in our previous studies were related to lesion size. This appears to be the case. The rats in the present study did not develop what are essentially microcephalic brains, as we found in our earlier studies. Nonetheless, we are still puzzled by the severe consequences of the lesions in our earlier studies because we have made similarly large lesions of the frontal or occipital cortex without such devastating sequelae. Again, it appears that there is something special about the posterior parietal region.

\section{Dendritic Growth After Cortical Lesions in Infancy}

We have shown repeatedly that when the brain sustains damage in infancy, there are compensatory changes in the extent of dendritic arborization and spine density (for a review, see Kolb, 1995). In all such studies, the presence of dendritic change was correlated with some restitution of function. In the present study we failed to find any dendritic growth and actually found some atrophy. There was also no functional recovery. This result is consistent with the general hypothesis that functional recovery occurs when there is synaptic reorganization in the remaining cortex and fails to occur when this reorganization does not occur. Because we have found dendritic growth after adult or infant lesions of the medial frontal cortex and after infant lesions of the motor or occipital cortex, the absence of dendritic growth in the present study is puzzling. Furthermore, when we have removed an entire hemisphere, including the PPC, in infancy we have seen compensatory growth in the intact hemisphere. Again, there appears to be something special about damage to the PPC.

We have hypothesized elsewhere that the reason that animals fare better after cortical injuries around 10 days is that this is during the time of maximal astrocyte production. Since astrocytes are presumed to produce factors that enhance neuron survival and differentiation, increased astrocyte proliferation could be expected to enhance functional outcome. In the present study we attempted to avoid opening the ventricle by removing only gray matter and leaving the underlying external capsule intact. Nonetheless, it is clear from the histological analysis that the ventricle was open in every brain. Since the astrocytes are likely being produced by stem cells in the ventricular zone, we cannot be certain that the ventricular perturbation is not interfering with astrocyte production. If so, this could account for the poorer dendritic growth and absence of functional recovery in the present experiment. We note, however, that ventricular opening after large frontal lesions does not prevent dendritic growth, although the ventricular disruption is in a different location than it was after the PPC lesions of the present study.

\section{Sex Differences in Spatial Navigation and Recovery of Function}

The study of sex differences in cognitive abilities of both laboratory animals and human neurological patients has been an active, if controversial, area of research for over 35 years (see, e.g., Clark \& Goldman-Rakic, 1989; Hampson \& Kimura, 1992; Kimura, 1992; Lansdell, 1962; McGlone, 1980; Roof, Zhang, Glasier, \& Stein, 1993). In the course of doing studies on the effects of medial frontal cortex lesions on spatial navigation in rats, we also began to notice that there are sex differences in frontal-operated rats that are not present in normal rats (e.g., Kolb \& Cioe, 1996; Kolb \& Stewart, 1995). These sex differences are correlated with sexually dimorphic differences in dendritic response to the lesion as well (e.g., Kolb \& Stewart, 1995). In contrast, we have not found sex differences in the effect of PPC lesions on behavior in either adult or infant operates, and there is also no apparent sex difference in dendritic response. These results are a bit surprising because Kimura (1992) has reported sex differences in the effects of both frontal and PPC lesions in humans. As we noted earlier, however, there is a fundamental difference in the visually guided behavior of primates and rodents, which may be important in this regard.

One result in the present study does bear particular comment. We found a sex difference in the performance of the Morris water task in both normal animals and in the P5 lesion animals. This result was surprising because 
although we found a similar result in a previous study as well (Kolb, Petrie, \& Cioe, 1996), we typically have not found such differences (e.g., Forgie \& Kolb, 1998; Kolb \& Cioe, 1996). There is one critical difference between the studies in which we have and have not found the differences, however. We have found a sex difference only in studies in which the normal animals have been anesthetized with hypothermia neonatally (see also Nuñez, Kim, McFarland, \& Juraska, 1997). This leads us to the conclusion that neonatal cooling may have effects upon the brain and behavior of rats, and in particular of female rats. (This idea was originally suggested to us by Janice Juraska.) One possibility is that the treatment is stressful and that it is the stress that is responsible for the sex difference, but this seems unlikely since the literature suggests that males are more affected by perinatal stress than are females (e.g., Fleming, Anderson, Rhees, Kinghorn, \& Bakaitis, 1986; Stewart \& Kolb, 1988). Nonetheless, whatever the explanation, the phenomenon is worthy of further study.

\section{REFERENCES}

BAYER, S. A., \& Altman, J. (1987). Direction in neurogenetic gradients and patterns of anatomical connections in the telencephalon. Progress in Neurobiology, 29, 57-106.

Clark, A. S., \& Goldman-RakIC, P. S. (1989). Gonadal hormones influence the emergence of cortical function in nonhuman primates. Behavioral Neuroscience, 103, 1287-1295.

Coleman, P. D., \& Riesen, A. H. (1968). Environmental effects on cortical dendritic fields: I. Rearing in the dark. Journal of Anatomy, 102, 363-374.

DiMATtia, B. V., \& KeSNER, R. P. (1988a). Role of the posterior parietal association cortex in the processing of spatial event information. Behavioral Neuroscience, 102, 397-403.

DiMatTia, B. V., \& Kesner, R. P. (1988b). Spatial cognitive maps: Differential role of parietal cortex and hippocampal formation. Behavioral Neuroscience, 102, 471-480.

Fleming, D. E., Anderson, R. H., Rhees, R. W., Kinghorn, E., \& BAKAITIS, J. (1986). Effects of prenatal stress on sexually dimorphic asymmetries in the cerebral cortex of the male rat. Brain Research Bulletin, 16, 395-398.

FORGIE, M., \& KOLB, B. (1998). Sex differences in the effects of frontal cortex injury: Role of differential hormonal experience in early development. Behavioral Neuroscience, 112, 141-153.

Galaburda, A. M. (1993). Neurology of developmental dyslexia. Current Opinion in Neurobiology, 3, 237-242.

GibB, R., \& KolB, B. (1998). A vibratome method for Golgi-Cox staining. Journal of Neuroscience Methods, 79, 1-4.

Hampson, E., \& Kimura, D. (1992). Sex differences and hormonal influences on cognitive function in humans. In J. B. Becker, S. M. Breedlove, \& D. Crews (Eds.), Behavioral endocrinology (pp. 357398). Cambridge, MA: MIT Press.

Kesner, R. P., Farnsworth, G., \& DiMattia, B. V. (1989). Double dissociation of egocentric and allocentric space following medial prefrontal and parietal cortex lesions in the rat. Behavioral Neuroscience, 103, 956-961.

KimURA, D. (1992, September). Sex differences in the brain. Scientific American, 267(3), 118-125.

KoLB, B. (1995). Brain plasticity and behavior. Mahwah, NJ: Erlbaum. Kolb, B., Buhrmann, K., McDonald, R., \& Sutherland, R. J. (1994). Dissociation of the medial prefrontal, posterior parietal, and posterior temporal cortex for spatial navigation and recognition memory in the rat. Cerebral Cortex, 6, 664-680.

KolB, B., \& ClOE, J. (1996). Sex-related differences in cortical function after medial frontal lesions in rats. Behavioral Neuroscience, 110, 1271-1281.

Kol., B., \& Giвв, R. (1993). Possible anatomical basis for recovery of function after neonatal frontal lesions in rats. Behavioral Neuroscience, 107, 1-13.

KolB, B., Holmes, C., \& Whishaw, I. Q. (1987). Recovery from early cortical lesions in rats: III. Neonatal removal of posterior parietal cortex has greater behavioral and anatomical effects than similar removals in adulthood. Behavioural Brain Research, 26, 119-137.

KolB, B., LADOWSKy, R., GibB, R., \& GoRNy, G. (1995). Does dendritic growth underlie recovery from neonatal occipital lesions in rats? $B e$ havioural Brain Research, 77, 125-133.

Kolb, B., Petrie, B., \& CIOE, J. (1996). Recovery from early cortical lesions in rats: VII. Comparison of the behavioural and anatomical effects of medial prefrontal lesions at different ages of neural maturation. Behavioural Brain Research, 79, 1-13.

KolB, B., \& STEWART, J. (1995). Changes in neonatal gonadal hormonal environment prevent behavioral sparing and alter cortical morphogenesis after early frontal cortex lesions in male and female rats. Behavioral Neuroscience, 109, 285-294.

Kolb, B., Sutherland, R. J., \& Whishaw, I. Q. (1983). A comparison of the contributions of the frontal and parietal association cortex to spatial localization in rats. Behavioral Neuroscience, 97, 13-27.

KOLB, B., \& WALKEY, J. (1987). Behavioural and anatomical studies of the posterior parietal cortex in the rat. Behavioural Brain Research, 23, 127-145.

KolB, B., \& Whishaw, I. Q. (1985). Earlier is not always better: Behavioural dysfunction and abnormal cerebral morphogenesis following neonatal cortical lesions in the rat. Behavioural Brain Research, $17,25-43$.

KolB, B., \& WhishaW, I. Q. (1989). Plasticity in the neocortex: Mechanisms underlying recovery from early brain damage. Progress in Neurobiology, 32, 235-276.

LANSDELl, H. A. (1962). A sex difference in effect of temporal-lobe neurosurgery on design preference. Nature, 194, 852-854.

MCGLONE, J. (1980). Sex differences in human brain asymmetry: A critical survey. Behavioral \& Brain Sciences, 3, 215-263.

Milner, D., \& GoOdale, M. (1995). The visual brain in action. Oxford: Oxford University Press.

MoRRIs, R. G. M. (1981). Spatial localization does not require the presence of local cues. Learning \& Motivation, 12, 239-260.

Nuñez, J. L., KıM, B. Y., McFarland, E. E., \& JuRaska, J. M. (1997). Effect of neonatal cryoanesthesia on the gross size of the adult rat cortex. Society for Neuroscience Abstracts, 22, 62.

Roof, R. L., Zhang, Q., Glasier, M. M., \& Stein, D. G. (1993). Gender-specific impairment on Morris water maze task after entorhinal cortex lesion. Behavioural Brain Research, 57, 47-51.

SHOLL, D. A. (1956). The organization of the cerebral cortex. London: Methuen.

STEWART, J., \& KolB, B. (1988). The effects of neonatal gonadectomy and prenatal stress on cortical thickness and asymmetry in rats. Behavioral \& Neural Biology, 49, 344-360.

Sutherland, R. J., Whishaw, I. Q., \& KolB, B. (1983). A behavioural analysis of spatial localization following electrolytic, kainate- or colchicine-induced damage to the hippocampal formation in the rat. Behavioural Brain Research, 7, 133-153.

UNGERLEIDER, L. G., \& MishKin, M. (1982). Two cortical visual systems. In D. J. Ingle, M. A. Goodale, \& R. J. W. Mansfield (Eds.), Analysis of visual behavior (pp. 549-586). Cambridge, MA: MIT Press.

Whishaw, I. Q., O'Connor, W. T., \& DunnetT, S. B. (1986). Contributions of motor cortex, caudate-putamen, and nigrostriatal systems to skilled forepaw use in the rat. Brain, 109, 805-843.

Whishaw, I. Q., \& Tomie, J. (1989). Olfaction directs skilled forelimb reaching in the rat. Behavioural Brain Research, 32, 11-21.

ZILLES, K. (1985). The cortex of the rat: A stereotaxic atlas. Berlin: Springer-Verlag.

(Manuscript received August 7, 1997; revision accepted for publication December $8,1997$. ) 